\title{
Algorithm for Fast Calculation of Hirzebruch-Jung Continued Fraction Expansions to Coding of Graph Manifolds
}

\author{
Fernando I. Becerra López, Vladimir N. Efremov, Alfonso M. Hernández Magdaleno \\ Department of Mathematics, CUCEI, University of Guadalajara, Guadalajara, México \\ Email: mail@ferdx.com, efremov@cencar.udg.mx, 137mag@gmail.com
}

Received 6 August 2015; accepted 5 September 2015; published 8 September 2015

Copyright (C) 2015 by authors and Scientific Research Publishing Inc.

This work is licensed under the Creative Commons Attribution International License (CC BY). http://creativecommons.org/licenses/by/4.0/

c) (i) Open Access

\section{Abstract}

We present a new algorithm for the fast expansion of rational numbers into continued fractions. This algorithm permits to compute the complete set of integer Euler numbers of the sophisticate tree graph manifolds, which we used to simulate the coupling constant hierarchy for the universe with five fundamental interactions. Moreover, we can explicitly compute the integer Laplacian block matrix associated with any tree plumbing graph. This matrix coincides up to sign with the integer linking matrix (the main topological invariant) of the graph manifold corresponding to the plumbing graph. The need for a special algorithm appeared during computations of these topological invariants of complicated graph manifolds since there emerged a set of special rational numbers (fractions) with huge numerators and denominators; for these rational numbers, the ordinary methods of expansion in continued fraction became unusable.

\section{Keywords}

Hirzebruch-Jung Continued Fraction, Fast Expansion Algorithm, Graph Manifolds

\section{Introduction}

Hirzebruch-Jung (H-J) continued fractions are widely used in various branches of mathematics as well as in theoretical physics. First of all, HJ-continued fractions arise naturally in the minimal resolution of cyclic quotient (that is, Hirzebruch-Jung) surface singularities of the type $\mathbb{C} / \mathbb{Z}_{p}$, which is also known as HJresolution [1] [2]. This type of resolution gives rise to a smooth 4-dimensional manifold $X(p, q)$ called HJspace [3], which is homeomorphic to a plumbing graph manifold $P\left(\Gamma_{p}\right)$ described in [4] [5]. The boundary of HJ-space $X(p, q)$ is lens space $L(p, q)$, which can be also obtained as a link of Hirzebruch-Jung surface 
singularity $\mathbb{C} / \mathbb{Z}_{p}$. HJ-continued fractions are used also to describe the plumbing decomposition of the other type of link of surface singularities, namely, Seifert fibered homology spheres (Sfh-spheres), particularly Brieskorn homology spheres (Bh-spheres) [4] [6] [7].

In condensed matter theory, the HJ-continued fractions are used to describe fractional quantum Hall (FQH) systems with $k$ levels of hierarchy [8]-[10]. The main numerical characteristic of FQH effect in topological fluids is the filling factor, which is naturally presented in form of a HJ-continued fraction. Also, in topological string theory, the structure of internal space (Calabi-Yau threefold) can be encoded in terms of HJ-continued fraction expansion of positive integers, which are the topological invariants of the internal space and define the mode of interactions between D-branes [3] [11].

Recently, we have attempted to use the plumbing procedure and corresponding HJ-continued fraction expansion to solve the problems of the coupling constant hierarchy [5] [12] [13] and fine tuning [14] on the base of Kaluza-Klein approach in the multidimensional topological field theory, where plumbing graph manifolds play the role of internal spaces. In the Section 2 we shall explain these ideas. In this way a set of special rational numbers (irreducible fractions) with huge numerators and denominators arise. For these rational numbers, the ordinary methods to find their expansion in continued fraction are impractical due to the number of operations involved; thus in Section 3 we develop an algorithm for fast expansion of HJ-continued fraction. This gives us the possibility to evaluate the number of vertexes of plumbing graphs which can be used to explain the coupling constant hierarchy and the rank of the corresponding Laplacian block matrices [5]. In Section 4, we give an example of calculation of a special continued fraction by means of our algorithm.

\section{Continued Fractions and Graph Manifolds}

In this section, we review the main concepts of codifying the topology of plumbing graph manifolds by means of continued fraction expansion (for more detales see [1] [2] [5]). For given coprime integers $p$ and $q$, the Hirzebruch-Jung modification of euclidean algorithm

$$
\left(\lambda_{i}, \lambda_{i+1}\right)\left(\begin{array}{ll}
n_{i} & 1 \\
-1 & 0
\end{array}\right)=\left(\lambda_{i-1}, \lambda_{i}\right), n_{i} \in \mathbb{N} \backslash\{1\} ; i=1, \cdots, k,
$$

leads to HJ-continued fraction

$$
\frac{p}{q}=\left[n_{1}, n_{2}, \cdots, n_{k}\right]:=n_{1}-\frac{1}{n_{2}-\frac{1}{\cdots-\frac{1}{n_{k}}}},
$$

if we set the "initial" conditions to $\lambda_{0}=p, \lambda_{1}=q$ (this expansion is unique under supposition of continued fraction finiteness). The expansion process is finished for $k$ such that $\lambda_{k}=1, \lambda_{k+1}=0$. By means of product

$$
\left(\begin{array}{ll}
n_{1} & 1 \\
-1 & 0
\end{array}\right) \cdots\left(\begin{array}{cc}
n_{k} & 1 \\
-1 & 0
\end{array}\right)=\left(\begin{array}{ll}
p & q \\
-q^{*} & p^{*}
\end{array}\right) .
$$

Fr. Hirzebruch defined in [1] the plumbing procedure which leads to the 4-dimensional plumbing manifold $P\left(\Gamma_{p}\right)$ with the boundary $\partial P\left(\Gamma_{p}\right)=L(p, q)$. The plumbing manifold is also known as a graph manifold corresponding to tree graph $\Gamma_{p}$ (of type $A_{k}$ ) shown in Figure 1.

With the aim of obtain the coupling constant hierarchy we consider tree plumbing graphs $\Gamma_{p}$ of more general type [5] [13], whose basic structure blocks are Seifert fibered Brieskorn homology spheres [7]. Let $a_{1}, a_{2}, a_{3}$ be pairwise relatively prime positive numbers, the Brieskorn homology sphere (Bh-sphere) is defined as the link of Brieskorn singularity

$$
\begin{aligned}
& \Sigma(\underline{a}):=\Sigma\left(a_{1}, a_{2}, a_{3}\right):=\left\{z_{1}^{a_{1}}+z_{2}^{a_{2}}+z_{3}^{a_{3}}=0\right\} \cap S^{5} . \\
& \dot{n}_{1} \quad \dot{n}_{2} \quad \cdots \overrightarrow{n_{k}}
\end{aligned}
$$$$
\text { Figure 1. The tree graph } \Gamma_{p} \text { of type } A_{k} \text {. }
$$ 
Bh-spheres belong to the class of Seifert fibered homology spheres (Sfh-spheres). On each of these manifolds, there exists a Seifert fibration with unnormalized Seifert invariants $\left(a_{i}, b_{i}\right)$ subject to

$$
e(\Sigma(\underline{a}))=\sum_{i=1}^{3} b_{i} / a_{i}=-1 / a,
$$

where $a=a_{1} a_{2} a_{3}$ and $e(\Sigma(\underline{a}))$ is its rational Euler number, the well known topological invariant of a Bh-sphere. If we define continued fraction expansions $\frac{a_{i}}{b_{i}}=\left[n_{i, 1}, \cdots, n_{i, k_{i}}\right](i=1,2,3)$ then the plumbing graph corresponding to Bh-spheres $\Sigma\left(a_{1}, a_{2}, a_{3}\right)$ is given in Figure 2 [1].

We begin the construction of the principal ensemble of Bh-spheres with definition of a primary sequence (for details see [12]). Let $p_{i}$ be the ith prime number in the set of positive integers $\mathbb{N}$, i.e. $p_{1}=2, p_{2}=3, \cdots, p_{9}=23, \cdots$, then the primary sequence of Bh-spheres is defined as

$$
\left\{\Sigma\left(p_{2 n}, p_{2 n+1}, q_{2 n-1}\right) \mid n \in \mathbb{Z}^{+}\right\},
$$

where $q_{i}:=p_{1} \cdots p_{i}$. The first terms in this sequence with $n>0$ (which we really use in this section) are $\Sigma(2,3,5)$ (the Poincaré homology sphere), $\Sigma(7,11,30), \Sigma(13,17,2310)$, and $\Sigma(19,23,510510)$. We also include in this sequence as its first term the manifold $\Sigma(1,2,1)$, corresponding to $n=0$ with the following definition $p_{0}=q_{-1}=1$, i.e. the usual three-dimensional sphere $S^{3}$ with Seifert fibration (Sf-sphere).

Instead of proceeding with the general consideration (which is rather large and has been realized in [13] [14]) we want to take up a simplified example, that illustrates in what manner the rational number closed to the experimental value of cosmological constant might appear in our construction. We start considering the term with $n$ $=4$ in the primary sequence, that is $\Sigma(19,23,510510)$, that has the rational Euler number (i.e. the $1 \times 1$ rational linking matrix $K^{11}$ )

$$
e(\Sigma(19,23,510510))=K^{11}=\frac{-5}{19}+\frac{-3}{23}+\frac{200933}{510510}=\frac{1}{223092870} \approx 4.48 \times 10^{-9} .
$$

Here we use the classical expression for the rational Euler invariant $e\left(\Sigma\left(a_{1}, a_{2}, a_{3}\right)\right)=\sum_{i=1}^{3} b_{i} / a_{i}$, where $b_{i}$ are unnormalized Seifert invariants $\left|b_{i}\right|<a_{i}$, defined uniquely by $b_{i} a / a_{i}=1 \bmod a_{i}$ ( $b_{i}$ and $a_{i}$ are coprime integeres). Thus, $b_{1}=-5, b_{2}=-3, b_{3}=200933$. More details in [7].

Now we consider the derivative of Bh-sphere [12] defined as

$$
D^{(1)} \Sigma(\underline{a})=D^{(1)} \Sigma\left(a_{1}, a_{2}, a_{3}\right):=\Sigma\left(a_{1}, a_{2} a_{3}, a+1\right),
$$

where we can see the result of this operation is the Bh-sphere with Seifert invariants

$$
a_{1}^{(1)}=a_{1}, a_{2}^{(1)}=a_{2} a_{3}, a_{3}^{(1)}=a+1
$$

and the Euler invariant $e\left(D^{(1)} \Sigma(\underline{a})\right)=1 / a^{(1)}$, where $a^{(1)}=a_{1}^{(1)} a_{2}^{(1)} a_{3}^{(1)}=a(a+1)$. Applying the derivate successively, we define by induction the $m$-th derivative $D^{(m)} \Sigma(\underline{a})=\Sigma\left(a_{1}^{(m)}, a_{2}^{(m)}, a_{3}^{(m)}\right)$ for any $m \in \mathbb{N}$. Calculating 4-th derivative to the Bh-sphere $\Sigma(19,23,510510)$, we obtain the Bh-sphere $D^{(4)} \Sigma(19,23,510510)$ with Euler number $(1 \times 1$ rational linking matrix $)$

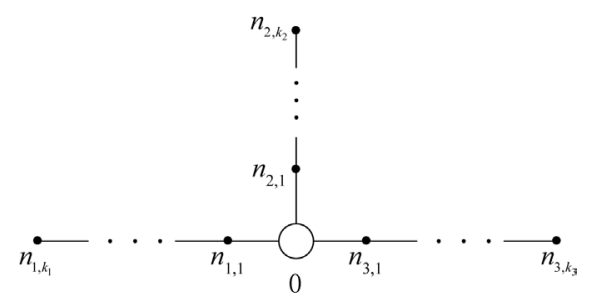

Figure 2. The plumbing graph corresponding to Bh-spheres $\Sigma\left(a_{1}, a_{2}, a_{3}\right)$. 


$$
\begin{aligned}
e^{(4)}= & K^{(4) 11}=\frac{-5}{19}+\frac{849861850484706836195974405889499139290451730552325577772918123429}{322947503184188597754470274238009672930371657609883719537088869030} \\
& +\frac{-1}{6136002560499583357334935210522183785677061494587790671204688511571}
\end{aligned}
$$$$
-0 . \overline{263157894736842105}
$$$$
+0.2631578947368421052631578947368421052631578947368421052631578947370 \ldots
$$$$
-1.629725525927064843 \times 10^{-67} \approx 2.66 \times 10^{-134} \text {. }
$$

The consequences of these calculations are following. It is possible to interpret the rational linking $(1 \times 1)$ matrix $K^{(4) 11}$ (formed by the unique rational Euler number $e^{(4)}$ ) as a coupling constant of the unique interaction, "switched on" in the universe owing to the presence of three-dimensional internal space, namely, the Bh-sphere $D^{(4)} \Sigma(19,23,510510)$ in Kaluza-Klein approach [13]. By its numerical value, $2.66 \times 10^{-134}$, this constant may be identify with the cosmological constant in the contemporary universe. Also in [13] we argue that the constant $K^{11} \approx 4.48 \times 10^{-9}$, that is the rational Euler number of the Bh-sphere $\Sigma(19,23,510510)$, can be associated with a cosmological constants in the universe at the Planck scale of time. Thus the ratio $K^{(4) 11} / K^{11}$ might characterize the contemporary cosmological constant in Planck density units. Note that even by these crude estimations, we have $\Lambda=K^{(4) 11} / K^{11} \approx 5.94 \times 10^{-126}$, that is less than the empirical bound [15] [16]

$$
\rho_{\Lambda}=(1.35 \pm 0.15) \times 10^{-123},
$$

only on three orders. Moreover, the calculations of Euler numbers by the Formulas (6) and (8) lead to the conclusion that the absolute value of each summand is many orders larger that the resulting Euler number which represents the cosmological constant. This fact simulates the fine tuning effect in the modeling scheme, which we have proposed in [14].

At this point, it emerges the problem of calculate integer Euler numbers $\left\{n_{i, s_{i}} \mid s_{i}=1, \cdots, k_{i}, i=1,2,3\right\}$, which correspond to the plumbing graphs of type shown in Figure 2 which describe Bh-spheres, or equivalently, to calculate the Laplacian matrix for the plumbing graph [5]. (Note that the set of integer Euler numbers is the most important topological invariant of any tree graph manifold.) This problem is reduced to calculate the continued fraction expansions for all rational numbers $\frac{a_{i}}{b_{i}}=\left[n_{i, 1}, \cdots, n_{i, k_{i}}\right](i=1,2,3)$ in the algebraic sum which forms the rational Euler number of a graph manifold. As we can see in the example (8) the second fraction has the huge numerator and denominator, so ordinary methods of expansion in continued fraction represent a lot of operations (as it was mentioned in the Introduction). Note that the same situation occurs for more sophisticated tree graph manifolds which we have to use to describe the coupling constant hierarchy in the universe real [14]. The fact is that our universe contains at least five fundamental interactions (strong, electro-magnetic, weak, gravitational and cosmological) with the corresponding coupling constants. In our model [13] each coupling constant is connected with the rational Euler number of proper Bh-sphere, so to obtain five coupling constants it is necessary to paste together (by plumbing) five certain Bh-spheres. The graph corresponding to this situation is shown in Figure $3^{1}$. Specific examples are given in [13] [14]. The characteristic feature of all these schemes is the emergence (in the structure of rational Euler invariants) of irreducible fractions with enormous numerators and denominators, which continued fraction expansions are too large to be obtained by classical methods. As an example, in Section 4 we work with the "greatest" fraction which emerges in [14] (Formula (5.12))

$$
\frac{q_{1}}{p_{1}}=
$$

5224868486304546518657

32059806410238279718139966062484464209573437243152889136180581555726013473961031111880160 ,

${ }^{1}$ In Figure 3 the integer Euler numbers are denoted as $\varepsilon_{m}^{I}$ and $e_{n}^{I}$ and $N^{I}$ is $I$-th node (vertex with the valence 3). The Euler number corresponding to any node is 0 , since the unnormalized Seifert invariants are used. More details see in [5]. 


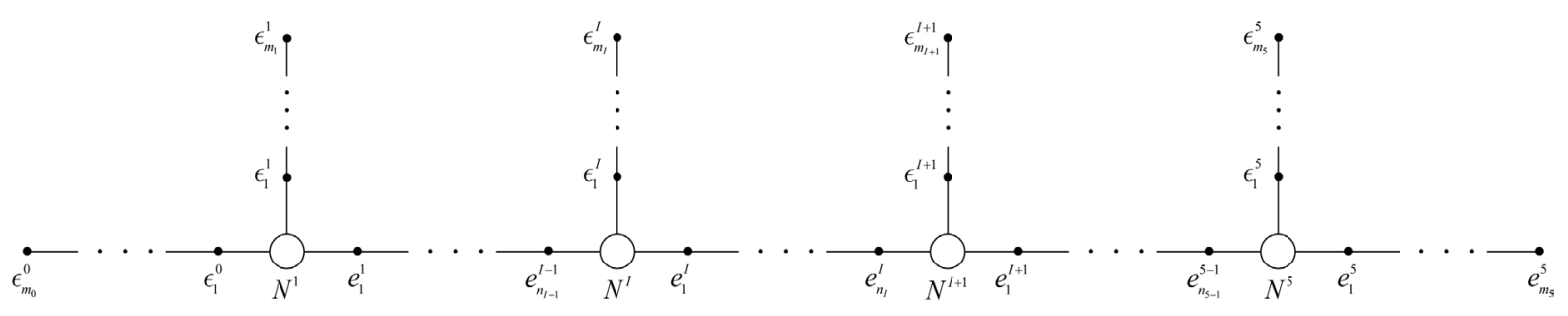

Figure 3. The plumbing graph corresponding to the plumbing of five certain Bh-spheres.

whose continued fraction expansion is represented by $\approx 4 \times 10^{20}$ terms, and shows the importance of the fast calculation algorithm we have created and that we state in the following section. Note that in the case of plumbing of Brieskorn homology spheres, which is under consideration in our paper (see Figure 3), the explicit formulas derived by Saveliev in [4], ensure that the integers $p_{1}$ and $q_{1}$ are coprimes.

\section{Algorithm to Obtain Continued Fraction Expansion}

First of all, let's analyze the fraction $\frac{x}{x-r}$, where $x, r \in \mathbb{N}$. The quotient of this fraction will be 1 if

$$
\frac{x}{x-r}<2 \Rightarrow x>2 r
$$

and a direct calculation shows that

$$
\frac{x}{x-r}=2-\frac{x-2 r}{x-r}=2-\frac{1}{\frac{x-r}{x-r-r}}=2-\frac{1}{2-\frac{1}{\frac{x-r-r}{x-r-r-r}}},
$$

where we observe the difference between the numerator and denominator keeps constant, and so we can repeat the process $k$ times until obtain the fraction $\frac{x-k r}{x-(k+1) r}$ where $x-(k+1) r<r$, which means the quotient won't be 1 and we have to restart the process until we get a quotient 1 . Moreover, due to condition (10), we can write $x=2 r+y \quad(y \in \mathbb{N})$; so

$$
\frac{x}{x-r}=\frac{2 r+y}{r+y}=2-\frac{1}{\frac{r+y}{y}},
$$

and for the last fraction to satisfy condition (10) we need $y>r$. In this case we can repeat the process

$$
\frac{r+y}{y}=2-\frac{1}{\frac{y}{y-r}},
$$

and now we need $y>2 r$ to get the condition satisfied. So, we have to find the minimum $k$ such that $y<k r$, i.e.

$$
k=\left\lceil\frac{y}{r}\right\rceil=\left\lceil\frac{x-2 r}{r}\right\rceil=\left\lfloor\frac{x-r}{r}\right\rfloor
$$

Now, suposse we want the continued fraction expansion for $\frac{p}{q}$, with $p$ and $q$ coprimes and $p, q \in \mathbb{N}, p>q$. We can observe that this fraction can be rewritten as 


$$
\frac{p}{q}=\frac{p}{p-(p-q)}
$$

that fits with the decomposition in (11) if $p<2 q$, so under this case, we can reduce the process obtaining the minimum number $k=\left\lfloor\frac{q}{p-q}\right\rfloor$ as in (13). Looking at this result, we can work fractions with $p>2 q$ (note that $p=2 q$ implies the fraction is indeed integer), repeating the process until we obtain the condition $p<2 q$ and then find $k$ that is the number of quotients equal to 1 . Moreover, we can reduce even more the process if we note that the fraction $\bar{k}=\left\lfloor\frac{q}{p \bmod q}\right\rfloor$ includes the value of $k$ besides the number of quotients different to 1 obtained of the previous decomposition to obtain a quotient 1 . This is because $p \bmod q$ represents the value of $r$ in (11) and then $\bar{k}$ indicates the number of times we have to repeat the decomposition until not having the condition $x>2 r$.

We can obtain all the coefficients of the expansion with these results. Every time we get a quotient 1 , we take the value of $\bar{k}$ to calculate the number of quotients 1 will appear until the condition $p<2 q$ is not satisfied and thus we will get a quotient different to 1 . In other words, $s=\left\lceil\frac{p}{q}\right\rceil$ gives us the quotient we need to continue the expansion and $\bar{k}=\left\lfloor\frac{q}{p \bmod q}\right\rfloor$ gives us the number of terms of the decomposition until we get $s>2$ again. Also, we can expand the fraction

$$
\frac{p}{q}=\frac{(s-1) q+r}{q}=s-\frac{1}{\frac{q}{q-r}},
$$

where we note the similarity between the last fraction and (11), so $q \bmod r$ will be the denominator of the fraction where condition (10) is not satisfied. So, we have an iterative procedure that avoid the direct calculation of all these quotients and remainders.

Finally, let $\frac{p_{1}}{q_{1}}$ be the original fraction, we can summarize the process in the next algorithm:

$$
\begin{aligned}
& \text { Require: } p_{1}>q_{1} \\
& i=0 \\
& \text { repeat } \\
& i=i+1 \\
& r_{i}=p_{i} \bmod q_{i} \\
& s_{i}=\left[\frac{p_{i}}{q_{i}}\right] \\
& \bar{k}_{i}=\left[\frac{q_{i}}{r_{i}}\right] \\
& q_{i+1}=q_{i} \bmod r_{i} \\
& p_{i+1}=q_{i+1}+r_{i} \\
& \text { until } r_{i}=1 \text { or } q_{i+1}=1 \\
& \text { if } r_{i}=1 \text { then } \\
& \frac{p_{1}}{q_{1}}=[s_{1}, \underbrace{2, \cdots, 2}_{\bar{k}_{1}-1 \text { times }}, s_{2}, \underbrace{2, \cdots, 2}_{\bar{k}_{2}-1 \text { times }}, \cdots, s_{i}, \underbrace{2, \cdots, 2}_{\bar{k}_{i}-1 \text { times }}] \\
& L=\sum_{j=1}^{i} \bar{k}_{j} .
\end{aligned}
$$


end if

if $q_{i+1}=1$ then

$$
\begin{aligned}
& \frac{p_{1}}{q_{1}}=[s_{1}, \underbrace{2, \cdots, 2}_{\bar{k}_{1}-1 \text { times }}, s_{2}, \underbrace{2, \cdots, 2}_{\overline{k_{2}}-1 \text { times }}, \cdots, s_{i}, \underbrace{2, \cdots, 2}_{\bar{k}_{i}-1 \text { times }}, p_{i+1}] \\
& L=1+\sum_{j=1}^{i} \bar{k}_{j} .
\end{aligned}
$$

end if

In the case $q_{i+1}=1$ a last term $p_{i+1}$ has to be added to the expansion. In the algorithm, $L$ represents the length of the expansion for $\frac{p_{1}}{q_{1}}$, which is obtained from every $s_{i}$ and a chain of $\bar{k}_{i}-1$ terms equal to 2 .

\section{Using of the Algorithm to Calculate a Very Long Expansion}

Now we show the benefits of the algorithm calculating a special (and long) continued fraction expansion. The algorithm allow us to know the length of expansions even if the number of coefficients is such that it is impossible to write, by giving us the number of terms equal to 2 in the expansion. Let's take coprime integers

$$
p_{1}=
$$

32059806410238279718139966062484464209573437243152889136180581555726013473961031111880160,

$q_{1}=5224868486304546518657$ (fraction obtained from the study of rational linking matrix of graph manifolds described in Section 2). We calculate

$$
\begin{aligned}
& r_{1}=p_{1} \bmod q_{1}=13, \\
& s_{1}=\left\lceil\frac{p_{1}}{q_{1}}\right\rceil=6136002560499583357334935210522183785677061494587790671204688511572, \\
& \bar{k}_{1}=\left\lfloor\frac{q_{1}}{r_{1}}\right\rfloor=401912960484965116819, \\
& q_{2}=q_{1} \bmod r_{1}=10 \\
& p_{2}=q_{2}+r_{1}=23,
\end{aligned}
$$

because $r_{1} \neq 1, q_{2} \neq 1$, we do

$$
\begin{aligned}
& r_{2}=p_{2} \bmod q_{2}=3, \\
& s_{2}=\left\lceil\frac{p_{2}}{q_{2}}\right\rceil=3, \\
& \bar{k}_{2}=\left\lfloor\frac{q_{2}}{r_{2}}\right\rfloor=3, \\
& q_{3}=q_{2} \bmod r_{2}=1,
\end{aligned}
$$

where we stop because $q_{3}=1$. So, the expansion for this fraction can be written as

[6136002560499583357334935210522183785677061494587790671204688511572, 2, ․, 2, 3, 2, 2, 4]

where the suspension points represent 401912960484965116818 numbers 2 of the same number of fractions 
whose quotient is 1 . So, the lenght of the continued fraction which represents $\frac{p_{1}}{q_{1}}$ is

$$
L=1+\sum_{j=1}^{2} \bar{k}_{j}=1+401912960484965116819+3=401912960484965116823 .
$$

This results clearly shows the benefits of the algorithm against the classical method of the remainders. To illustrate this, the fastest computer over the world at the time, Tianhe- $2^{2}$, would need $\approx 3$ hours to get this expansion.

\section{Conclusions}

The main result of this article is an algorithm for the fast expansion of rational numbers into HJ-continued fractions. This algorithm gives us the possibility to calculate (with a few operations) the complete set of integer Euler numbers (the principal topological invariant) of sophisticate graph manifolds, which we used to simulate the coupling constant hierarchy of the fundamental interactions acting in our universe [13]. Automatically we can explicitly compute the integer Laplacian block matrix associated with any tree plumbing graph [5]. This matrix coincides up to sign with the integer linking matrix of the graph manifold corresponding to the plumbing graph [14]. The rational linking matrix (describing the coupling constants hierarchy) can be obtained from the Laplacian one by means of Gauss-Neumann partial diagonalization procedure described explicitly in [5] [17]. It makes sense to emphasize that the rank of the integer Laplacian block matrix, corresponding to the realistic model coupling constants hierarchy is of order $10^{20}$. This gives an idea of the numerical array capacity which is used to simulate the coupling constants hierarchy in the universe, containing five fundamental interactions.

We can make an important physical assumption that the tridiagonal submatrices

$$
K^{a b}(\underline{e}, I)=\left(\begin{array}{cccc}
e_{1}^{I} & -1 & & \\
-1 & \ddots & & \\
& & \ddots & -1 \\
& & -1 & e_{n_{I}}^{I}
\end{array}\right) ; \quad K^{\alpha \beta}(\underline{\varepsilon}, I)=\left(\begin{array}{cccc}
\varepsilon_{m_{I}}^{I} & -1 & & \\
-1 & \ddots & & \\
& & \ddots & -1 \\
& & -1 & \varepsilon_{1}^{I}
\end{array}\right)
$$

of the integer Laplacian block matrix, corresponding to a plumbing graph shown in Figure 3, describe the lowenergy physics of hierarchical topological fluids with $n_{I}$ and $m_{I}$ levels respectively [8] [10], which are put together according to the structure of the plumbing graph. Thus our universe can be represented as the set of hierarchically organized BF system of topological fluids (more details see in [5] [14]). It is important to note the analogy between our model and the description of superconductivity based on the topological defects in terms of BF systems [18], where continued fractions also play a significant role.

\section{References}

[1] Hirzebruh, F. (1971) Differentiable Manifolds and Quadratic Forms. Marcel Dekker, New York.

[2] Popescu-Pampu, P. (2007) The Geometry of Continued Fractions and the Topology of Surface Singularities. Advanced Studies in Pure Mathematics, 46.

[3] Griguolo, L., Seminara, D., Szabo, R.J. and Tanzini, A. (2007) Black Holes, Instanton Counting on Toric Singularities and q-Deformed Two-Dimensional Yang-Mills Theory. Nuclear Physics B, 772, 1-24. http://dx.doi.org/10.1016/j.nuclphysb.2007.02.030

[4] Saveliev, N. (2002) Fukomoto-Furuta Invariants of Plumbed Homology 3-Spheres. Pacific Journal of Mathematics, 205, 465-490. http://dx.doi.org/10.2140/pjm.2002.205.465

[5] Becerra, F., Efremov, V. and Hernandez, A. (2014) Block Matrix Representation of a Graph Manifold Linking Matrix Using Continued Fractions. Applied Mathematics, 5, 1894-1902. http://dx.doi.org/10.4236/am.2014.513183

[6] Neumann, W. (1981) A Calculus for Plumbing Applied to the Topology of Complex Surface Singularities and Degenerating Complex Curves. Transactions of the American Mathematical Society, 268, 299-344. http://dx.doi.org/10.1090/S0002-9947-1981-0632532-8

\footnotetext{
2“TOP \#1 SYSTEMS” at Top500 The List, consulted August 5th, 2015. http://www.top500.org/featured/top-systems/
} 
[7] Saveliev, N. (2002) Invariants for Homology 3-Spheres. Springer, Berlin. http://dx.doi.org/10.1007/978-3-662-04705-7

[8] Wen, X.G. and Zee, A. (1992) Classification of Abelian Quantum Hall States and Matrix Formulation of Topological Fluids. Physical Review B, 46, 2290. http://dx.doi.org/10.1103/PhysRevB.46.2290

[9] Balachandran, A.P., Chandar, L. and Sathiapalan, B. (1995) Chern-Simons Duality and Quantum Hall Effect. Nuclear Physics B, 443, 465-500. http://dx.doi.org/10.1016/0550-3213(95)00122-9

[10] Fujita, M., Li, W., Ryu, S. and Takayanagi, T. (2009) Fractional Quantum Hall Effect via Holography: Chern-Simons, Edge States, and Hierarchy. Journal of High Energy Physics, 2009, JHEP06. http://dx.doi.org/10.1088/1126-6708/2009/06/066

[11] Harvey, J.A., Kutasov, D., Martinec, E.J. and Moore, G. (2001) Localized Tachyons and RG Flows. arXiv: hep-th/0111154v2.

[12] Efremov, V.N., Mitskievich, N.V., Hernandez Magdaleno, A.M. and Serrano Bautista, R. (2005) Topological Gravity on Plumbed V-Cobordism. Classical and Quantum Gravity, 22, 3725. http://dx.doi.org/10.1088/0264-9381/22/17/022

[13] Efremov, V.N., Hernandez Magdaleno, A.M. and Moreno, C. (2010) Topological Origin of the Coupling Constants Hierarchy in Kaluza-Klein Approach. International Journal of Modern Physics A, 25, 2699. http://dx.doi.org/10.1142/S0217751X10048482

[14] Efremov, V., Hernandez, A. and Becerra, F. (2014) The Universe as a Set of Topological Fluids with Hierarchy and Fine Tuning of Coupling Constants in Terms of Graph Manifolds. arXiv:1309.0690v2.

[15] Tegmark, M., Aguirre, A., Rees, J. and Wilczek, F. (2006) Dimensionless Constants, Cosmology and Other Dark Matter. Physical Review D, 73, Article ID: 023505. http://dx.doi.org/10.1103/PhysRevD.73.023505

[16] Bousso, R. (2007) TASI Lectures on the Cosmological Constant. arXiv: hep-th/0708.4231.

[17] Neumann, W. (1997) Commensurability and Virtual Fibration for Graph Manifolds. Topology, 36, 355-378. http://dx.doi.org/10.1016/0040-9383(96)00014-6

[18] Diamantini, M.C. and Trugenberger, C.A. (2015) Higgsless Superconductivity from Topological Defects in Compact BF Terms. Nuclear Physics B, 891, 401-419. http://dx.doi.org/10.1016/j.nuclphysb.2014.12.010 